\title{
A Cell Specific Reference Signal Interference Cancellation Scheme for LTE Cellular Access Systems
}

\author{
Wei Li, Yue Zhang(Yue_Zhang@ieee.org), Li-ke Huang, John Cosmas, Qiang Ni
}

\begin{abstract}
Heterogeneous network is supported to improve the link performance in LTE Rel. 10+. The network introduces co-existence of Macro and Pico cell which can alleviate the potential traffic congestion caused by increased user density. Along with the co-existence of difference cells comes the disadvantage of inter-cell interference. Although almost blank subframe (ABS) is proposed to mitigate the interference, the reference signal in ABS still inevitably causes interference. This paper focuses on the interference cancellation by utilizing the information in the ABS. First, the interference signal model which takes account of channel effect, time and frequency error is presented. Based on this model, interference cancellation scheme is studied. The timing and carrier frequency offset of interference signal is compensated. Afterwards, the reference signal of interfering cell is generated locally and channel response is estimated by making use of channel statistics. Then, the interference signal is reconstructed based on previous estimate of channel, timing and carrier frequency offset. The interference is then mitigated by substracting the estimate of interference signal. Computer simulation shows this interference cancellation algorithm significantly improves performance in different channel conditions.
\end{abstract}

Index Terms-LTE, Cell edge interference, almost blank subframe, eICIC

\section{INTRODUCTION}

$\mathrm{H}$ eterogeneous network (HetNet) with macro and pico cell is introduced to meet the huge traffic demand and extend the coverage. In this network topology, the high power $(1 \sim 40 \mathrm{~W})$ macro cell provides basic coverage and the low power $(250 \mathrm{~mW})$ pico cell serves as complementary cell. The pico cell can functionally extend network coverage and off-load data traffic of macro-cell, which reduces cost and increase frequency efficiency. However, the user equipments UEs served by pico cell will also receive RF signal from neighbor high power macro cells. This issue is even server when users of the pico cell stay within the coverage of macro cells with range extension enabled [1].

In Rel. 10, Enhanced inter-cell interference coordination (eICIC) [2] is introduced to address this issue. eICIC proposes two technique. First, the signal strength is biased to pico-cell

It is the policy of the IEEE to own the copyright to the technical contributions it publishes on behalf of the interests of the IEEE, its authors, and their employers and facilitate the appropriate reuse of this material by others which reduces the interference power. Second, macro cell remains silent for certain periods, termed almost blank subframe (ABS) [3]. In the ABS, the physical downlink shared channel (PDSCH) is emptied. Hence, UE does not receive PDSCH during ABS and the interference can be alleviated. However, the users can still receive cell specific reference signal (CRS), paging channel (PCH), physical broadcast channel $(\mathrm{PBCH})$, and synchronization channels (PSS/SSS) which degrades the performance. Hence, further eICIC (FeICIC) is proposed in Release 11 to eliminate this CRS interference problem.

There exist few researches on CRS interference cancellation (IC). [16] and [17] investigate direct IC and LLR puncturing methods. The simulation results show that direct interference provides better performance. [18] proposes receiver algorithm that combines IC with direct decision channel estimation (CE) algorithm for colliding CRS. In [19], a space alternating generalized expectation-maximization (SAGE) with a maximum a-posteriori (MAP) method is proposed to estimate interfering channel, which reduces computation complexity compared with linear minimum mean square error (LMMSE) method. However, timing error and frequency offsets can severely degrade the performance.

In this paper, CRS interference cancellation algorithm for non-colliding scenario which makes use of channel statistics is investigated. First, the interference signal is analyzed and modeled. Further, the interference cancellation algorithm is discussed based on this model. The algorithm makes uses of primary synchronization signal (PSS) and secondary synchronization signal (SSS) to obtain timing offset (TO) and carrier frequency offset (CFO). Then, the channel response is estimated by utilizing channel statistics. Afterwards, the interference signal is reconstructed. Then, interference is alleviated by substracting interference signal from received signal.

The rest of this paper is organized as follows: The interference is analyzed and modeled in Section II. Section III discusses IC algorithms. The computer simulation is presented in Section IV and the conclusion is drawn in Section V.

\section{INTERFERENCE ANALYSIS AND MODEL}

Fig. 1 illustrates typical inter-cell interference scenario between macro and pico cells, in which the LTE service is 
delivered to the subscriber via pico cell and the downlink signal from macro cell interferes the UEs at the edge of pico cell. To alleviate the inter-cell interference, the ABS is transmitted by the macro cell, during which only certain control signal is

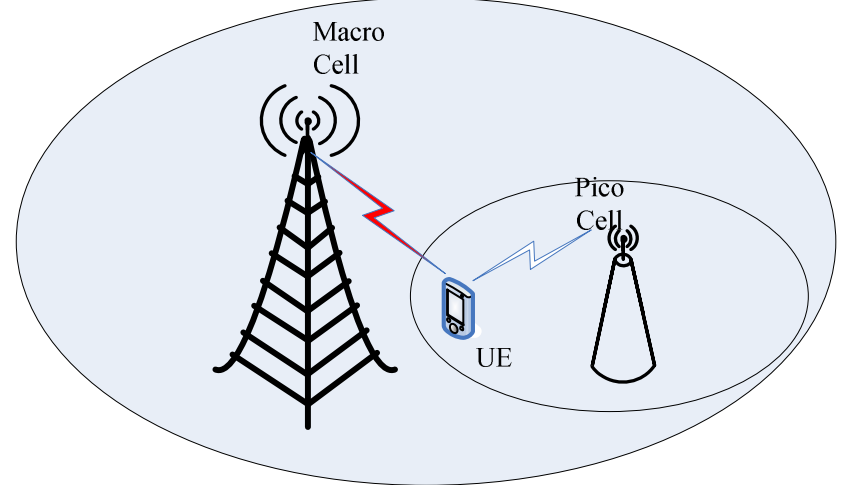

Fig. 1 Inter-cell interference between macro and pico cell

transmitted, such as CRS. However, the CRS still causes interference to the subscriber. The colliding scenario where the interference signal overlaps CRS REs of the serving cell is out of the scope of this paper. Fig.2 shows a non-colliding interference scenario. In this scenario, the received signal of one resource block (RB) with one interference cell. As shown in this figure, the CRS signal from neighbor macro cell overlaps the resource elements (REs) from serving cell (SC). The SC RE can be divided into data RE and CRS RE. Due to the TO and CFO between the interfering cell and subscriber, the received interfering signal suffers $\mathrm{TO}$ and $\mathrm{CFO}$ as shown in this figure.

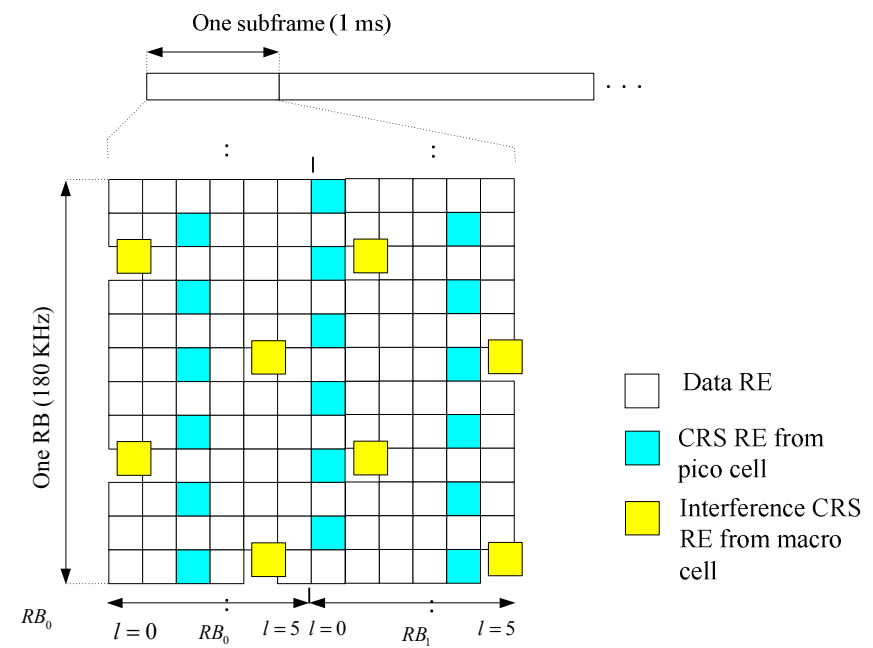

Fig. 2 Received signal in time and frequency grid

The LTE downlink transmission employs OFDM modulation, in which the frequency domain signal of $i^{t h}$ symbol is transferred to time domain signal $x_{i}(n)$ via $\mathrm{N}$-point Fast Fourier Transform (FFT):

$$
\begin{aligned}
x_{i}(n) & =\frac{1}{\sqrt{N}} \sum_{k=0}^{N-1} X_{i, k} e^{j 2 \pi n k / N} \\
& =\frac{1}{\sqrt{N}} \sum_{k=0}^{N-1}\left(d_{i, k}+p_{i, k}\right) e^{j 2 \pi n k / N}
\end{aligned}
$$

where $d_{i, k}$ and $p_{i, k}$ represents the data and pilot respectively.

Then the signal is transmitted over multipath propagation channel under consideration of additive white Gaussian noise (AWGN). At the receiver side, the received signal can be written as:

$$
y_{i}(n)=\sum_{l=0}^{L-1} h_{l} x_{i}\left(n-\tau_{l}\right)+w(n)
$$

where $h_{l}$ and $\tau_{l}$ are the gain and the delay of the $l^{t h}$ path, respectively; $n_{k}$ is the additive Gaussian noise. Due to the TO and $\mathrm{CFO}$, the corrupted receiver signal is:

$$
\begin{aligned}
y_{i}(n)= & y_{i}^{(0)}(n)+y_{i}^{(1)}(n)+w(n) \\
= & \frac{1}{\sqrt{N}} \sum_{k=0}^{N-1} H_{i, k}^{(0)} X_{i, k}^{(0)} e^{j 2 \pi n k / N}+ \\
& \frac{1}{\sqrt{N}} \sum_{k=0}^{N-1} H_{i, k}^{(1)} X_{i, k}^{(1)} e^{j 2 \pi(n-d)\left(k+f_{\Delta}\right) / N}+ \\
& +w(n)
\end{aligned}
$$

where $y_{0}^{(0)}(n)$ and $y_{0}^{(1)}(n)$ are the desired and interference signal respectively; $H_{i, k}^{(0)}$ and $H_{i, k}^{(1)}$ is the frequency response of the serving and interfering channel; $d$ and $f_{\Delta}$ is the relative timing and frequency offset between the macro and pico cell. After applying the N-point FFT, the OFDM symbol is given by [5]:

$$
\begin{aligned}
Y_{i, k} & =Y_{i, k}^{(0)}+Y_{i, k}^{(1)} \\
& =H_{i, k}^{(0)} X_{i, k}^{(0)}+\sum_{n=-N / 2}^{N / 2} e^{\frac{j 2 \pi n d}{N}} \cdot H_{i, n}^{(1)} X_{i, n}^{(1)} \Phi_{n}+W_{i, k}
\end{aligned}
$$

where $H_{i, k}^{(0)}$ and $H_{i, k}^{(1)}$ demote the channel coefficients of serving and interfering cell at $k^{t h}$ subcarrier respectively; $\Phi_{i}$ stands for the inter-carrier interference (ICI). During the ABS, only CRS signal from interfering cell overlaps the data REs of serving cell as shown in Fig. 2. The signal model at the data REs of the serving cell with interference can be written as:

$$
Y_{i, k}=H_{i, k}^{(0)} d_{i, k}^{(0)}+\sum_{n=-N / 2}^{N / 2} e^{\frac{j 2 \pi n d}{N}} H_{i, n}^{(1)} p_{i, n}^{(1)} \Phi_{n}+W_{i, k}
$$

According to (5), the relative timing offset $d$ between interfering and serving cell causes phase shift $e^{\frac{j 2 \pi k d}{N}}$ on $k^{t h}$ subcarrier. The terms $\Phi_{n}$ in (5) arises from CFO term $f_{\Delta}$, which results in inter-carrier interference (ICI). Hence, the $\mathrm{CFO}$ and FO need to be compensated. In addition, this model shows single-input single-output (SISO) antenna case only. The multiple-input multiple-output (MIMO) antenna case can be easily derived based on (5). However, the number of RE will increase since the number of interference CRS increases with number of antenna ports, which results in more severe 
interference [4]. These problems will be addressed in Section III.

\section{IC ALGORITHM}

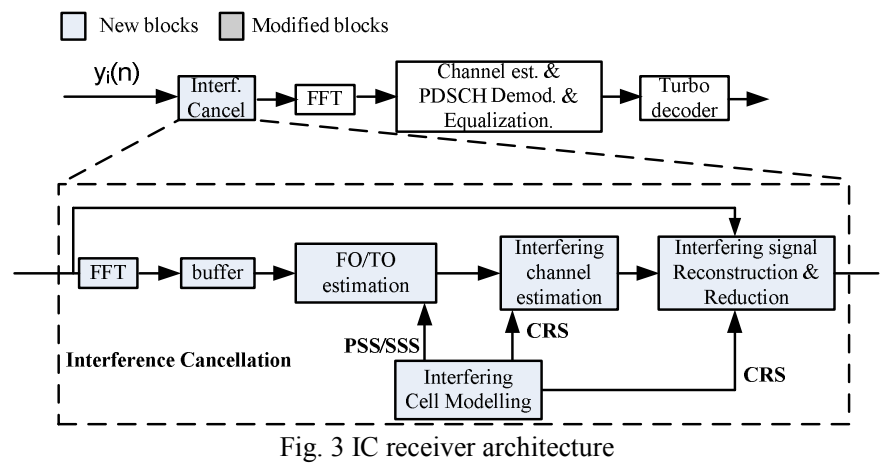

The inter-cell IC algorithm is proposed as shown in Fig. 3. Apart from the conventional LTE receiver modules, this algorithm mainly consists of the following parts: TO and CFO estimation, interfering channel estimation (CE), interfering cell modeling and interference signal reconstruction and reduction. In $\mathrm{CFO} / \mathrm{TO}$ estimation, the relative frequency offset and timing offset between the interfering cell and serving cell is estimated by using PSS/SSS generated in interfering cell modeling. Next, the interfering $\mathrm{CE}$ is conducted based on the compensated signal. The interfering signal is then reconstructed based on the previous estimation and subtracted from the received signal.

\section{A. CFO/TO Estimation}

The objective of this module is to retrieve OFDM symbol timing and estimate the CFO of the interfering cell. Many timing and frequency synchronization algorithm has been developed. Most of them exploits of the periodic nature of the time-domain signal by using cyclic prefix (CP) [6]-[8] or pilot data [9]-[10]. However, the data REs are zero in ABS, which significantly reduces the power of CP. The low SNR of CP makes it difficult for both timing and frequency synchronization. However, the PSS/SSS signal can be utilized for synchronization. The PSS and SSS are located at the last and second-last symbol in the time slot 0 and slot 10 . The PSS $p p p(n)$ and SSS $\operatorname{sss}(n)$ in frequency domain is given by:

$$
\operatorname{pss}(n)= \begin{cases}e^{\frac{j \pi u n(n+1)}{63}} & n=0,1, \cdots, 30 \\ e^{\frac{j \pi u(n+1)(n+2)}{63}} & n=31,32, \cdots, 61\end{cases}
$$

And

$$
\begin{gathered}
\text { sss }(2 n)= \begin{cases}s_{0}^{\left(m_{0}\right)}(n) c_{0}(n) \quad \text { time slot } 0 \\
s_{1}^{\left(m_{1}\right)}(n) c_{0}(n) \quad \text { time slot } 10\end{cases} \\
\operatorname{sss}(2 n+1)= \begin{cases}s_{1}^{\left(m_{1}\right)}(n) c_{1}(n) z_{1}\left(m_{0}\right)(n) & \text { time slot } 0 \\
s_{0}^{\left(m_{0}\right)}(n) c_{1}(n) z_{1}\left(m_{1}\right)(n) & \text { time slot } 10\end{cases}
\end{gathered}
$$

where $u$ is 25,29 or 34 corresponding to the physical layer cell identity $N_{I D}^{(2)} ; m_{0}$ and $m_{1}$ are derived from the physical layer cell identity group $N_{I D}^{(1)}$. Because the PSS/SSS symbol only contains PSS/SSS signal, which means the entire PSS/SSS symbol could be used for synchronization. As the time domain PSS/SSS symbol can make full use of CP and gives better performance, this paper adopts time domain synchronization method in [11]. The timing and frequency offset can be estimated by utilizing the cross-correlation of PSS/SSS symbol in time domain [11]:

$$
\left\{\hat{d}, \hat{f}_{\Delta}\right\}=\arg \max _{d, f_{\Delta}}\left(\left|C\left(d, f_{\Delta}\right)\right|\right)
$$

where

$$
C\left(d, f_{\Delta}\right)=\sum_{m=1}^{N / 2} s_{i}^{*}(n) r(n+m) e^{\frac{-2 \pi f_{\Delta^{n}}}{N}}
$$

And $r(n)$ and $s_{i}(n)$ are the received and locally generated symbol that contain PSS/SS.

Note that the generation of PSS/SSS is based on the assumption of ideal cell search. The cell search algorithm in scenario of inter-cell interference is beyond of the scope of this paper and will not be discussed. After the timing and frequency synchronization of the interference signal, the interfering channel response can be estimated.

\section{B. Interfering $C E$}

Before IC, it's essential to estimate the interfering channel response. The channel estimation can be based on Lease Square (LS) or Minimum Mean-Square (MMSE) [12], [13]. The MMSE algorithm is shown to give $10-15 \mathrm{~dB}$ gain in signal-to-noise ratio (SNR) for the same mean square error of CE over LS estimation [12]. However, the MMSE is more complex than the LS algorithm. After timing and frequency offset compensation, (5) can be rewritten as:

$$
Y_{i, k}=H_{i, k}^{(0)} d_{i, k}^{(0)}+H_{i, k}^{(1)} p_{i, k}^{(1)}+W_{i, k}
$$

The interfering CRS sequence $p^{(1)}$ can be expressed from the following equation:

$$
p^{(1)}=\frac{1}{\sqrt{2)}}(1-2 c(2 n))+j \frac{1}{\sqrt{2}}(1-2 c(2 n+1))
$$

where $c(n)$ is generated by a length-31 Gold sequence, the state of which is initialized according to the cell ID, slot number and antenna port. Assuming that the user conducts ideal cell search, the interfering CRS $p_{i, k}^{(1)}$ can be generated locally. Applying LS $\mathrm{CE}$, the estimation interfering channel can be written as:

$$
\hat{H}_{i, k}^{(1)}=Y_{i, k} / p_{i, k}^{(1)}=H_{i, k}^{(1)}+H_{i, k}^{(0)} d_{i, k}^{(0)} / p_{i, k}^{(1)}+W_{i, k} / p_{i, k}^{(1)}(11)
$$

According to (11), the data RE of serving cell $H_{i, k}^{(0)} d_{i, k}^{(0)} / p_{i, k}^{(1)}$ becomes interference whose power is relatively high. Hence, the estimate in (11) is not accurate. Numeric studies in [14] shows that the distribution of the interference signal is close to Gaussian for larger RB size and non-Gaussian for smaller RB size. And the mean of the distribution converges to 0 . Hence, the expectation of (11) can be derived as: 


$$
\begin{aligned}
\mathbb{E}\left\{\hat{H}_{i, k}^{(1)}\right\}= & \mathbb{E}\left\{Y_{i, k} / p_{i, k}^{(1)}\right\} \\
= & \mathbb{E}\left\{H_{i, k}^{(1)}+H_{i, k}^{(0)} d_{i, k}^{(0)} / p_{i, k}^{(1)}+W_{i, k} / p_{i, k}^{(1)}\right\} \\
= & \mathbb{E}\left\{H_{i, k}^{(1)}\right\}+\mathbb{E}\left\{H_{i, k}^{(0)} d_{i, k}^{(0)} / p_{i, k}^{(1)}\right\} \\
& +\mathbb{E}\left\{W_{i, k} / p_{i, k}^{(1)}\right\} \\
\approx & \mathbb{E}\left\{H_{i, k}^{(1)}\right\}
\end{aligned}
$$

(12) gives a good estimation of mean value of the interfering channel. The mean value of the interfering channel can be estimated by using a moving average window in the time dimension as shown in Fig. 4. If the moving average window length $\mathrm{M}$ is within the coherence time of the channel, $\hat{H}_{i, k}^{(1)}$ could be approximated by $\mathbb{E}\left\{\hat{H}_{i, k}^{(1)}\right\}$. The procedure of the interfering $\mathrm{CE}$ is show in Fig. 4:

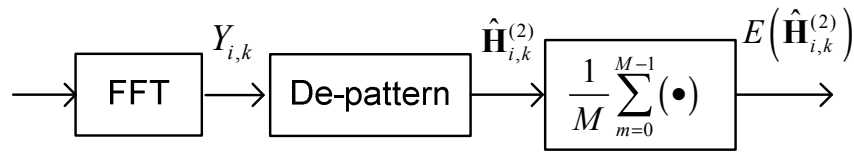

Fig. 4 Interfering CE

In MIMO and adjustable bandwidth scenario, the IC algorithm should be aware of the correct antenna number and bandwidth of the interference cell. However, this information is not available at the UE before UE decides to handover to that cell. Therefore, the interference cell antenna number and bandwidth need to be estimated at the UE.

This paper proposes a simple way that IC control block always set the maximum possible bandwidth and antenna, i.e. $20 \mathrm{MHz}$ and 4 antenna. Then, the interference cell modelling and interfering $\mathrm{CE}$ module estimates channel accordingly. If the actual bandwidth is smaller than $20 \mathrm{MHz}$, the power of the pilots outside the signal band will be to zero. The estimate of the channel that is out of the signal bandwidth is:

$$
\begin{aligned}
\mathbb{E}\left\{\hat{H}_{i, k, \text { out }}^{(1)}\right\}= & \mathbb{E}\left\{H_{i, k}^{(1)}\right\}_{\text {out }}+\mathbb{E}\left\{H_{i, k}^{(0)} d_{i, k}^{(0)} / p_{i, k}^{(1)}\right\}_{\text {out }} \\
& +\mathbb{E}\left\{W_{i, k} / p_{i, k}^{(1)}\right\}_{\text {out }} \\
\approx & 0+0+0
\end{aligned}
$$

(13) indicates that the estimate of the neighbour cell channel could filter out the interference and noise by moving average. So the power of the channel estimates is a reliable measurement to detect the signal bandwidth. The similar approach could be applied to the antenna number detection as well.

\section{Interfering Signal Reconstruction and Reduction}

After estimating CFO, TO and channel response, the estimated of interference signal can be reconstructed based on the local time domain CRS. The relative timing offset $d$ is potentially larger than the duration of $\mathrm{CP}$ which causes ISI within the OFDM window of desired signal. Hence, reconstructed frequency domain interference signal symbol by symbol could lead to inaccurate IC. This algorithm reconstructs the interference signal in time domain and substracts it from the received signal in time domain directly: where

$$
\hat{y}^{(0)}(n)=y(n)-\hat{y}^{(1)}(n+\hat{d}) e^{\frac{-j 2 \pi \hat{f}_{\Delta^{n}}}{N}} * \hat{h}_{l}
$$

$$
\hat{h}_{l}=F F T\left\{\hat{H}_{i}^{(1)}\right\}
$$

\section{Simulation RESUlts}

In this section, the performance of IC algorithm will be evaluated via Monte Carlo simulation. A typical two-cell interference scenario is simulated as shown in Fig. 1: The serving cell is set to work in MBSFN mode with $10 \mathrm{MHz}$ bandwidth and different modulation and coding schemes (MCS) to deliver the service, and the interfering cell transmits normal ABS with bandwidth of $5 \mathrm{MHz}$. During ABS, the CRS overlaps the data data RE of desired signal which causes inter-cell interference. The desired and interfering signal pass through time varying channel with a delay spread smaller than the duration of CP. In the simulation, the WINNER II C2 (EVA) [15] channel model with different Doppler frequency is adopted to investigate the IC performance in different channel conditions. The arriving time of desired and interfering signal is adjusted to evaluate the effect of relative timing offset. In addition, different $\mathrm{CFO}$ is applied to interfering signal to evaluate its effects. In order to generate correct PSS/SSS/CRS for IC, the user is assumed to conducts ideal cell search.

Fig. 5 (a)-(d) shows BLER performance versus signal-to-noise ratio (SNR) in different IC scenarios. The MCS 8 and MCS 16 are adopted. The signal is transmitted via EVA channel with $5 \mathrm{~Hz}$ Doppler frequency at different SNR. The antenna multiplex mode is set to SIMO and $2 \times 2$ MIMO. The block error rate (BLER) is select as the performance criteria, and it is calculated based on 10000 block transmissions. For comparison, the BLER of interference-free transmission is presented as a reference in blue curve. The figure also shows the performance with/without IC in red and grey curves respectively. According to the figure, the inter-cell interference causes performance degradation during the interested SNR range. The proposed IC algorithm can significantly improves the BLER performance which is nearly close to the interference-free case as the red curve shows in the figure.

Fig. 6 (a) - (d) shows the BLER performance of different Doppler frequency scenarios. The SIMO MCS 18 modulation is adopted in this simulation. The Doppler frequency varies from $5 \mathrm{~Hz}$ to $200 \mathrm{~Hz}$. The interference-free case is presented as a reference as shown in blue curve. The performance of interfering case and IC are shown in black and red curves respectively. According to Fig.6 (a)-(b), the performance of IC significantly improves the BLER performance for different SNR and Doppler frequencies which proves the robustness of the IC algorithm. 


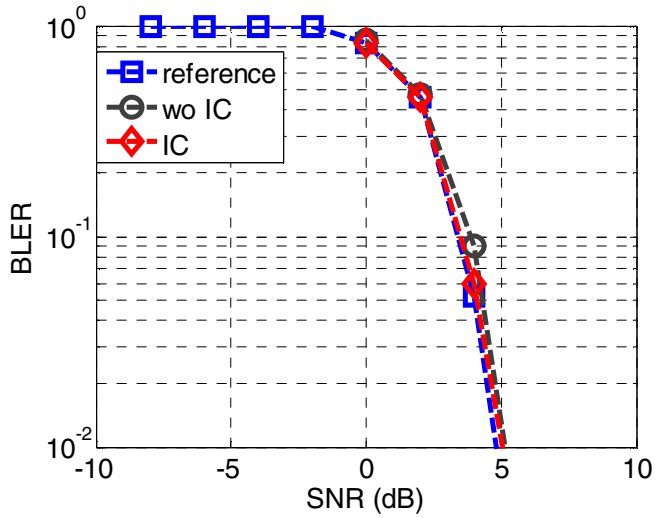

(a)

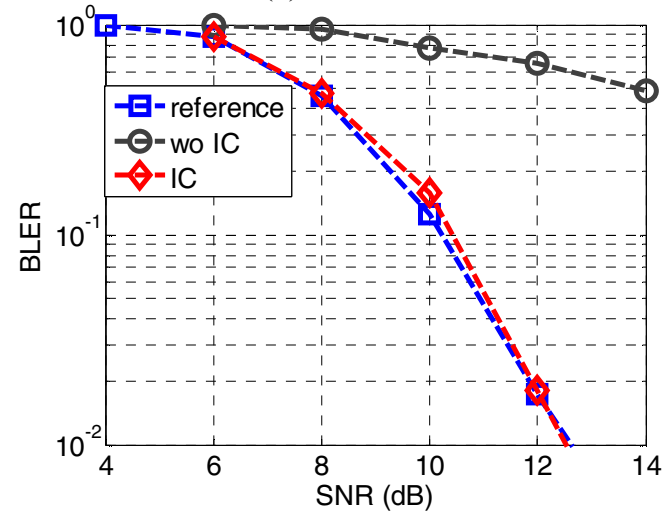

(c)

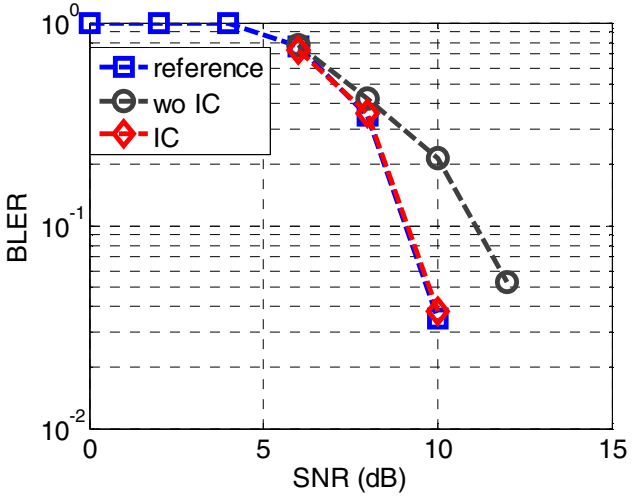

(b)

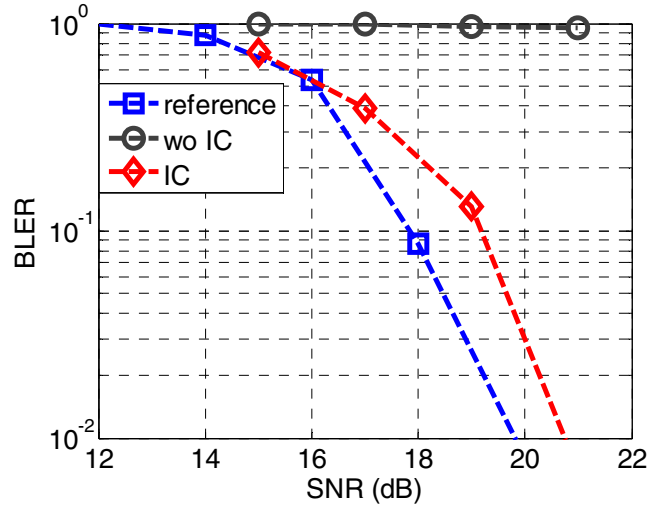

(d)

Fig. 5 BLER performance versus SNR in different IC scenarios: (a). SIMO, MCS 8; (b). SIMO, MCS 16; (c). MIMO, MCS 8; (d). MIMO, MCS 16

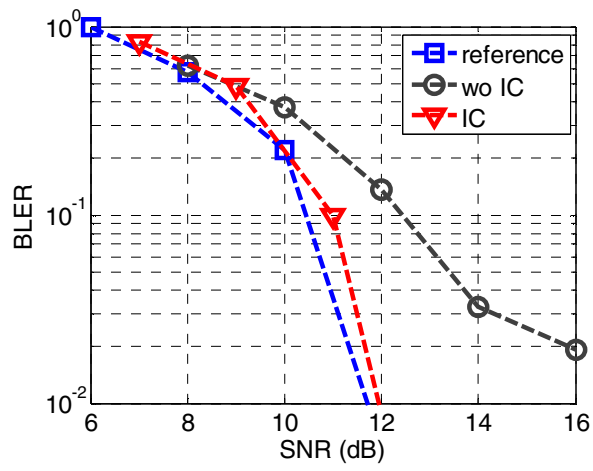

(a)

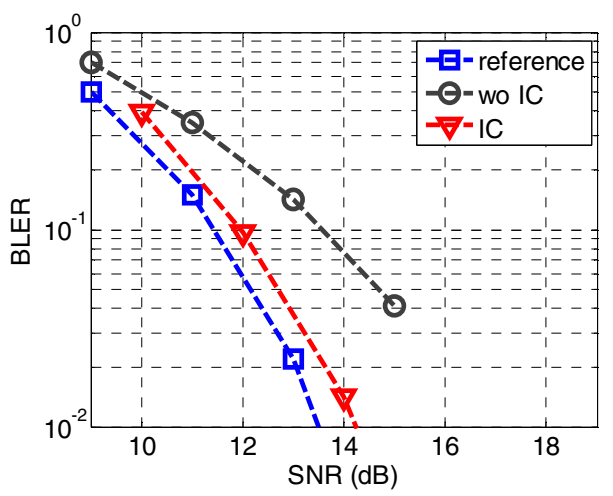

(c)

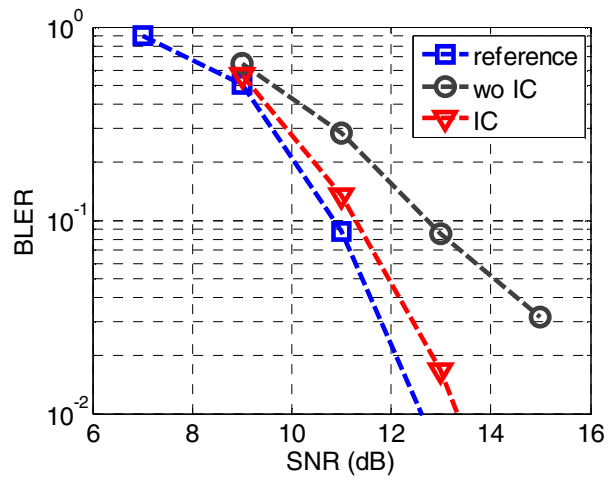

(b)

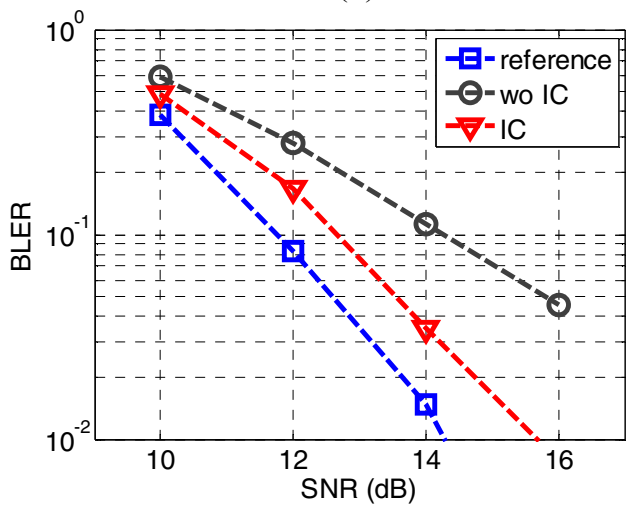

(d)

Fig. 6 BLER performance of different Doppler frequency scenarios: (a). $5 \mathrm{~Hz}$; (b). $70 \mathrm{~Hz}$; (c). $150 \mathrm{~Hz}$; (d). $200 \mathrm{~Hz}$ 


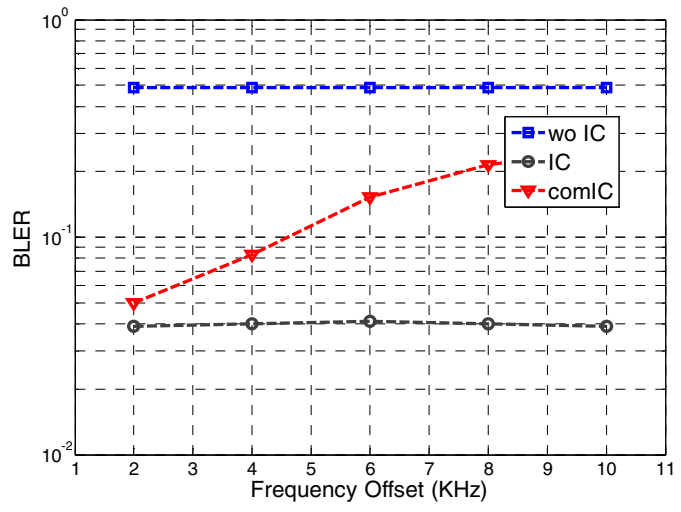

Fig. 7 BLER Performance vs. Frequency Offset

Fig. 7 shows the effect of CFO on the BLER performance. The performance of proposed IC algorithm and combined IC (comIC) algorithm in [18]. According to this figure, the performance of algorithm in [18] gradually degrades as the $\mathrm{CFO}$ increase. As contrary, there is no significant performance degradation for proposed algorithm. This proves the effectiveness of frequency synchronization method under when $\mathrm{CFO}$ is large.

Fig. 8 also show the effect of timing offset on the BLER performance. MCS 22 modulation is adopted. The channel is set to EVA $5 \mathrm{~Hz}$ and SNR is set to $16 \mathrm{~dB}$. The performance of proposed IC is shown in red curve. As a comparison, the combined IC (comIC) algorithm in [18] is shown in grey curve. The red curve shows that proposed IC achieves significantly improvement when there is small delay or very large delay (the inference pilot almost overlaps with the following symbol). When the delay is larger than half of OFDM symbol, the BLER of grey curve increase which means timing synchronization is required. The red curve shows that the IC with timing synchronization achieves robust performance within the interested timing offset range.

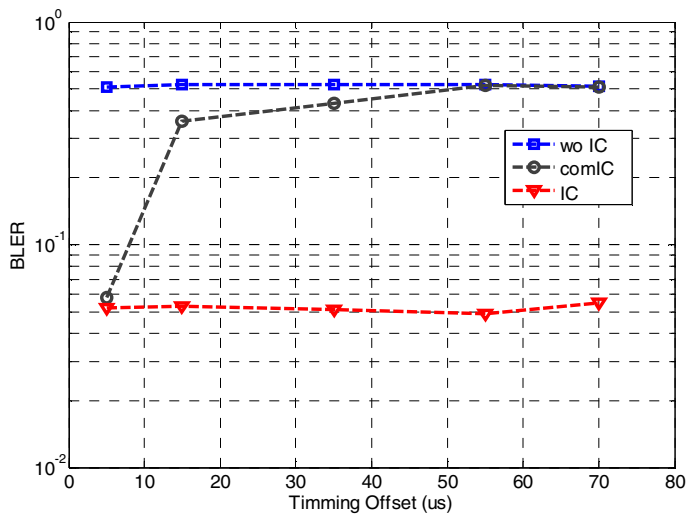

Fig. 8 BLER Performance vs. Timing Offset

\section{CONCLUSION}

This paper aims to cancel the inter-cell interference caused by the CRS in the ABS in cell edge. This paper firstly studies the signal model, which considers the interference signal from neighbor cell, channel effect, timing and frequency offset. Based on this model, the TO/CFO and interfering channel are estimated. The interfering signal is then reconstructed locally.
Finally, the interference is alleviated by substracting the reconstructed interference signal. The computer simulation shows this IC algorithm can achieve significant performance improvement in different channel conditions.

\section{REFERENCES}

[1] S. N. S. Kshatriya, S. Kaimalettu, S. R. Yerrapareddy, K. Milleth, and N. Akhtar, "On interference management based on subframe blanking in Heterogeneous LTE networks," in Communication Systems and Networks

[2] S. Deb, P. Monogioudis, J. Miernik, J.P. Seymour, "Algorithms for Enhanced Inter-Cell Interference Coordination (eICIC) in LTE HetNets". IEEE/ACM Trans. Networking, vol. 22, pp. 137- 150, Feb. 2014

[3] S. Deb, P. Monogioudis, J. Miernik, and J. P. Seymour, "Algorithms for Enhanced Inter-Cell Interference Coordination (eICIC) in LTE HetNets," Networking, IEEE/ACM Transactions on, vol. 22, pp. 137-150, 2014. (COMSNETS), 2013 Fifth International Conference on, 2013, pp. 1-7.

[4] 3GPP TS 36.211, "Evolved Universal Terrestrial Radio Access (E-UTRA); Physical channels and modulation" Feb. 2013.

[5] N.-L. Hung, L.-N. Tho and C. C. Ko "RLS-basedjoint estimation and tracking of channel response, sampling, and carrier frequency offsets for OFDM", IEEE Trans. Broadcast., vol. 55, pp.84 -94 2009

[6] J.-J. van de Beek, M. Sandell, and P. O. Börjesson, "ML estimation of time and frequency offset in OFDM systems," IEEE Trans. Signal Processing, vol. 45, pp. 1800-1805, July 1997.

[7] M. Speth, D. Daecke, and H. Meyr, "Minimum overhead burst synchronization for OFDM based broadband transmission," in Proc. Global Telecommun. Conf., Sydney, Australia, Nov. 1998, pp. $2777-2782$

[8] C. C. Ko , R. Mo and M. Shi "A new data rotation based CP synchronization scheme for OFDM systems", IEEE Trans. Broadcast., vol. 51, no. 5, pp.315 -321 2005

[9] J.-S. Baek and J.-S. Seo "Effective symbol timing recovery based on pilot-aided channel estimation for MISO transmission mode of DVB-T2 system", IEEE Trans. Broadcasting, vol. 56, no. 2, pp.193 -200 2010

[10] Xianbin Wang, T.T. Tjhung, Yiyang Wu, B. Caron, "SER performance evaluation and optimization of OFDM system with residual frequency and timing offsets from imperfect synchronization" IEEE Trans. Broadcasting, vol. 49, pp. 170-177, Jun. 2003.

[11] Yao-Hsien Tsai and Tzu-Hsien Sang, "A new timing synchronization and cell search procedure resistant to carrier frequency offsets for 3GPP-LTE downlink", Communications in China (ICCC), 2012 First IEEE International Conf. on, Pp. 334-338, Beijing, China. Aug. 2012

[12] J.-J. van de Beek, O. Edfors, M. Sandell, S. K. Wilson, and P. O. Borjesson, "On channel estimation in OFDM systems," in Proc. IEEE 45th Vehicular Technology Conf., Chicago, IL, Jul. 1995, pp. 815-819.

[13] S. P. Bingulac, "On the compatibility of adaptive controllers (Published Conference Proceedings style)," in Proc. 4th Annu. Allerton Conf. Circuits and Systems Theory, New York, 1994, pp. 8-16.

[14] Cheng Feng, Hongyu Cui, Meng Ma, Bingli Jiao, "On Statistical Properties of Co-channel Interference in OFDM Systems," IEEE Communication Letters, vol. 17, pp. 2328-2331, Oct. 2013

[15] 3GPP TS 36.101, "Evolved universal terrestrial radio access (E-UTRA), user equipment (UE) radio transmission and reception," Jul. 2013

[16] Qualcomm Inc, "Enabling communication in harsh interference scenarios," R4-102350, 3GPP-RAN WG4 AH\#10-03, Bratislava, July 2010.

[17] Qualcomm Inc, "Link level simulations for FeICIC with 9dB cell range expansion," R4-123313, 3GPP-RAN WG4 \#63, Prague, May 2012.

[18] M. Huang and W. Xu, "Macro-femto inter-cell interference mitigation for 3 GPP LTE-A downlink," In Proc. IEEE Wireless Comm. and Net. Conf, pp. 75-80, Apr. 12012.

[19] B.E.Priyanto, S. Kant, F. Rusek, S. Hu, J. Chen. C. Wugenshi, "Robust UE Receiver with Interference Cancellation in LTE Advanced Heterogeneous Network", In IEEE Vehicular Technology Conf. , Las Vegas, pp. 1-7, Sept. 20 